\title{
Performance Evaluation of Fuzzy Rule-Based Systems with Class Priority for Medical Diagnosis Problems
}

\author{
Tomoharu Nakashima \\ Osaka Prefecture University \\ Naka-ku Gakuen-cho 1-1, Sakai \\ Osaka 599-8531, Japan \\ nakashi@cs.osakafu-u.ac.jp \\ Gerald Schaefer \\ Aston University \\ Aston Triangle \\ Birmingham B4 7ET, U.K. \\ g.schaefer@aston.ac.uk
}

\author{
Yasuyuki Yokota \\ Osaka Prefecture University \\ Naka-ku Gakuen-cho 1-1, Sakai \\ saka 599-8531, Japan \\ yokota@ci.cs.osakafu-u.ac.jp \\ Hisao Ishibuchi \\ Osaka Prefecture University \\ Naka-ku Gakuen-cho 1-1, Sakai \\ Osaka 599-8531, Japan \\ hisaoi@cs.osakafu-u.ac.jp
}

\begin{abstract}
In this paper we examine the performance of fuzzy rule-based systems with classification priority for medical diagnosis problems. The assumption in this paper is that a classification priority is given a priori for each class in a pattern classification problem. Our fuzzy rulebased system consists of a set of fuzzy if-then rules that are automatically generated from a set of given training patterns. The consequent class of fuzzy if-then rules are decided based on the number of covered training patterns for each class. We apply the fuzzy classifier with class priority to two medical diagnosis problems: appendix diagnosis and breast cancer diagnosis, and compare its performance with that of a conventional fuzzy rule-based systems.
\end{abstract}

\section{INTRODUCTION}

While in the past fuzzy rule-based systems have been applied mainly to control problems [1], [3], more recently they have also been used in pattern classification problems. There are many approaches to the automatic generation of fuzzy if-then rules from numerical data for pattern classification problems [4]-[9].

Pattern classification research typically focusses on the minimisation of misclassification. However, in real world problems it is also necessary to consider differenct cases of misclassification. For example, in the case of a credit approval problem where the task is to decide whether to give an applicant a loan or not, if the applicant is not appropriate for the loan but was decided to be given a loan, the loss incurred by this misclassifcation is the financed money plus any interest. On the other hand, if the applicant would have been able to pay back but was not given the loan, the incurred loss by this misjudgement is just the amount of lost interest.

Let us consider another example in which different misclassification scenarios need to be taken into account. In medical diagnosis of cancer two kinds of misclassifications have to be considered. One is a misclassification of a benign tumor as malignant while the other is a malignant tumor mistakenly diagnosed as benign. Obviously, the number of occurances of both misclassification cases should be as small as possible. However, it is obvious that the latter case (malignant tumor diagnosed as benign) is more serious than the first case (benign tumor diagnosed as malignant).

In order to handle situations such as the ones mentioned the concept of misclassification cost can be introduced [10]. Several researchers have developed algorithms that consider classification cost in the construction of classification systems. For example, Domingos [11] proposed MetaCost which converts error-based classification systems into cost-sensitive ones. In MetaCost, multiple error-based classification systems are generated from multiple sets of resampled training patterns with replacement. The final classification for an unknown pattern is made by voting among the generated classifiers. MetaCost can be viewed as a wrapper approach to costsensitive pattern classification because it can be applied to any classification system.

There is still a problem that often cost-sensitive classification is performed under the assumption that misclassification costs are given numerically. In real world problems however, it poves difficult to estimate these numerical costs. Thus most cost-sensitive learning algorithms cannot be applied to many real world problems.

In order to overcome this problem, we employ an assumption that can be more applicable to such problems, namely that only the classification priority is given for each class. For the example of medical diagnosis, the correct identification of malignant tumors is more important than that of benign ones. Expressed differently, the classification priority for malignant cases is higher than that of benign cases so that the misclassification of malignant tumors as benign is minimised even though the other misclassifications may increase.

In this paper we will evaluate the effectiveness of fuzzy rule-based systems with classification priority for two 


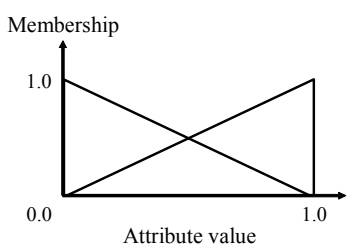

(a) Two fuzzy sets

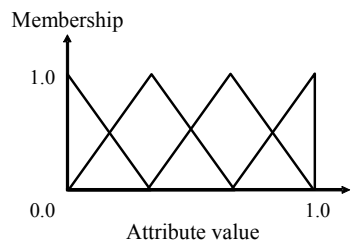

(c) Four fuzzy sets

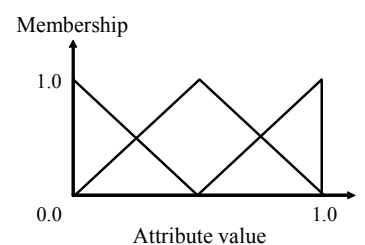

(b) Three fuzzy sets

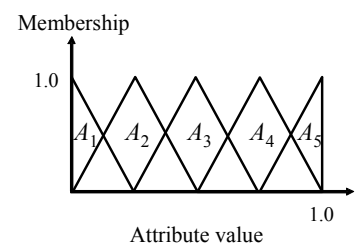

(d) Five fuzzy sets
Fig. 1. An example of antecedent fuzzy sets.

medical diagnosis problems. The reminder of the paper is organised as follows: First we explain conventional fuzzy rule-based systems in Section II. Next, the description of fuzzy rule-based systems with classification priority is provided in Section III. We the examine the performance of the fuzzy rule-based systems for the medical diagnosis problems in Section IV while we conclude the paper with Section V.

\section{Conventional Fuzzy Classification System}

In the literature various methods have been proposed for fuzzy classification [12]-[17]. In this paper we use the fuzzy-rule generation method proposed by Ishibuchi et al. [14] as the basis of our classification system.

\section{A. Pattern Classification Problems}

Let us assume that our pattern classification problem is an $n$-dimensional problem with $C$ classes and $m$ given training patterns $\mathbf{x}_{p}=\left(x_{p 1}, x_{p 2}, \ldots, x_{p n}\right), p=$ $1,2, \ldots, m$. Without loss of generality each attribute of the given training patterns is normalised into a unit interval $[0,1]$. That is, the pattern space is an $n$-dimensional unit hypercube $[0,1]^{n}$.

We use fuzzy if-then rules of the following type as basis of our classification system:

$$
\begin{array}{r}
\text { Rule } R_{j} \text { : If } x_{1} \text { is } A_{j 1} \text { and } \ldots \text { and } x_{n} \text { is } A_{j n} \\
\text { then Class } C_{j} \text { with } C F_{j}, \\
j=1,2, \ldots, N,
\end{array}
$$

where $R_{j}$ is the label of the $j$-th fuzzy if-then rule, $A_{j 1}, \ldots, A_{j n}$ are antecedent fuzzy sets on the unit interval $[0,1], C_{j}$ is the consequent class (i.e. one of the $C$ given classes), $C F_{j}$ is the grade of certainty of the fuzzy if-then rule $R_{j}$, and $N$ is the total number of rules. As antecedent fuzzy sets we use triangular sets as in Figure 1 where we show various partitions of the unit interval into a number of fuzzy sets.

\section{B. Generating Fuzzy If-Then Rules}

In our classification systems, we specify the consequent class and the grade of certainty of each fuzzy if-then rule from the given training patterns [14]. In [18] it is shown that the use of the grade of certainty in fuzzy if-then rules allows us to generate comprehensible fuzzy rulebased classification systems with high classification performance.

The consequent class $C_{j}$ and the grade of certainty $C F_{j}$ of fuzzy if-then rules are determined in the following manner:

\section{[Generation Procedure of Fuzzy If-Then Rule]}

Step 1: Calculate $\beta_{h}\left(R_{j}\right)$ for Class $h(h=1,2, \ldots, C)$ as

$$
\beta_{h}\left(R_{j}\right)=\sum_{\mathbf{x}_{p} \in \text { Class } h} \mu_{j 1}\left(x_{p 1}\right) \cdot \ldots \cdot \mu_{j n}\left(x_{p n}\right),
$$

where $\beta_{h}\left(R_{j}\right)$ is the sum of the compatibility of training patterns from Class $h$ with $R_{j}$, and $\mu_{j i}(\cdot)$ is the membership function of the fuzzy set $A_{j i}$.

Step 2: Find Class $\hat{h}$ that has the maximum value of $\beta_{h}\left(R_{j}\right)$ :

$$
\beta_{\hat{h}}\left(R_{j}\right)=\max \left\{\beta_{1}\left(R_{j}\right), \ldots, \beta_{C}\left(R_{j}\right)\right\}
$$

If two or more classes take the maximum value, the consequent class $C_{j}$ of the rule $R_{j}$ can not be determined uniquely. In this case, specify $C_{j}$ as $C_{j}=\phi$.

Step 3: If a single class takes the maximum value (i.e. if $\left.C_{j} \neq \phi\right)$, let $C_{j}$ be Class $\hat{h}$ and specify the grade of certainty $C F_{j}$ as

$$
C F_{j}=\frac{\beta_{\hat{h}}\left(R_{j}\right)-\bar{\beta}}{\sum_{h=1}^{C} \beta_{h}\left(R_{j}\right)},
$$

where

$$
\bar{\beta}=\frac{\sum_{h \neq \hat{h}} \beta_{h}\left(R_{j}\right)}{c-1} .
$$

The number of fuzzy if-then rules depends on how each attribute is partitioned into fuzzy sub-sets. For example, when we divide each attribute into three fuzzy sub-sets in a ten-dimensional pattern classification problem, the total number of fuzzy if-then rules is $3^{10}=59049$. The grade of certainty $C F_{j}$ can be adjusted by a learning algorithm [10].

\section{Fuzzy Reasoning}

Using the rule generation procedure outlined above we can generate fuzzy if-then rules as in (1). After both the consequent class $C_{j}$ and the grade of certainty $C F_{j}$ are determined for all $N$ rules, a new pattern $\mathbf{x}$ is classified 
by the following procedure:

[Fuzzy Reasoning Procedure for Classification]

Step 1: Calculate $\alpha_{h}(\mathbf{x})$ for Class $h, h=1,2, \ldots, C$, as

$$
\alpha_{h}(\mathbf{x})=\max \left\{\mu_{j}(\mathbf{x}) \cdot C F_{j} \mid C_{j}=\text { Class } h\right\},
$$

where

$$
\mu_{j}(\mathbf{x})=\mu_{j 1}\left(x_{1}\right) \cdot \ldots \cdot \mu_{j n}\left(x_{n}\right) .
$$

Step 2: Find Class $h^{\prime}$ that has the maximum value of $\alpha_{h}(\mathbf{x})$ :

$$
\alpha_{h^{\prime}}(\mathbf{x})=\max \left\{\alpha_{1}(\mathbf{x}), \ldots, \alpha_{C}(\mathbf{x})\right\} .
$$

If two or more classes take the maximum value, then the classification of $\mathbf{x}$ is rejected (i.e. $\mathbf{x}$ is left as an unclassifiable pattern), otherwise assign $\mathbf{x}$ to Class $h^{\prime}$.

\section{Proposed Fuzzy Classification}

In this section we describe a fuzzy rule-generation method for constructing cost-sensitive fuzzy classification systems that explicitly utilise costs of training patterns in the rule-generation process.

The assumption in this paper is that a classification priority is given a priori together with a set of training patterns. In the generation of fuzzy if-then rules, first we count the number of covered training patterns by a fuzzy if-then rule for each class. A training pattern is covered by a rule if the compatibility of the training pattern with the rule is larger than zero. Then the consequent class is determined as the class with the highest priority that has at least one covered training patterns.

As in the last section let us assume that we have $m$ training patterns $\mathbf{x}_{p}, p=1,2, \ldots, m$ for an $n$-dimensional $C$-class pattern classification problem. We also assume that a classification priority $r_{c}$ is given a priori for Class $c$, $c=1,2, \ldots, C$, where $r_{c}$ is a natural number and the classification priority decreases as the value increases. The procedure of generating a fuzzy if-then rule $R_{j}$ with class priorities $r_{c}, c=1,2, \ldots, C$, is summarized as follows:

[Generation Procedure of Fuzzy If-Then Rule]

Step 1: Count the number of covered training patterns $n_{c}^{j}$ from Class $c$ by the $j$-th fuzzy if-then rule $R_{j}$. The $p$-th training pattern $\mathbf{x}_{p}$ is covered by $R_{j}$ if the following equation holds:

$$
\mu_{j}\left(\mathbf{x}_{p}\right)>0.0
$$

where

$$
\mu_{j}\left(\mathbf{x}_{p}\right)=\mu_{j 1}\left(x_{p 1}\right) \cdot \mu_{j 2}\left(x_{p 2}\right) \cdot \ldots \cdot \mu_{j n}\left(x_{p n}\right) .
$$

Step 2: Find Class $\hat{h}$ that has the highest classification priority among the class with $n_{c}^{j}>0$ :

$$
\hat{h}=\arg \min _{c}\left\{r_{c} \mid n_{c}^{j}>0, c=1,2, \ldots, C\right\} .
$$

Step 3: Specify the grade of certainty $C F_{j}$ as

$$
C F_{j}=\frac{\beta_{\hat{h}}\left(R_{j}\right)}{\sum_{h=1}^{C} \beta_{h}\left(R_{j}\right)} .
$$

We modified the specification of the degree of certainty in (12) as the conventional specification in (4) makes the degree of certainty negative in some cases.

The fuzzy reasoning procedure for classifying unseen patterns is exactly the same as the conventional fuzzy rule-based classification described in Subsection II-C.

\section{Computational Experiments}

\section{A. Two-dimensional synthetic problem}

First we show the effect of introducing classification priority for a two-dimensional synthetic pattern classification problem shown in Figure 2 where 1,000 circle patterns (o) are uniformly distributed in the unit space $[0,1]^{2}$ except in the subspace $[0,0.1]^{2}$. On the other hand, square patterns $(\square)$ are normally distributed with a mean vector of $(0,0)$ and a variance of $0.1^{2}$ for both attributes. It is assumed that the classification priority of square patterns is higher than that of circle patterns.

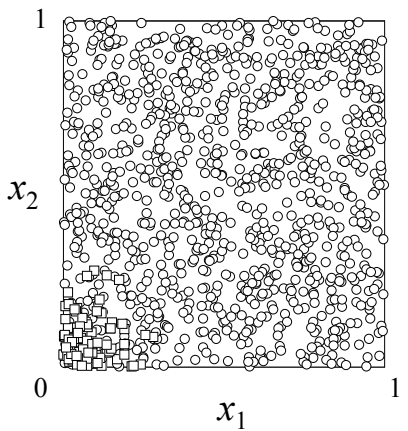

Fig. 2. Two-dimensional synthetic problem.

We examined the classification boundaries obtained from applying our proposed method by changing the number of fuzzy partitions for each attribute and compare it then to the boundaries generated by the conventional fuzzy rule-based systems described in Section II.

The obtained classification boundaries by the proposed method and the conventional method are shown in Figure 3 and Figure 4 respectively. We can see that all square patterns are correctly classified by the proposed method for all the four fuzzy partitions in Figure 3. We can also see from Figure 4 that all square patterns are misclassified when the number of fuzzy partitions is two ( $L=2$ ) although the number of misclassification decreases as the number of fuzzy partitions increases. The classification priority is hence successfully incorporated in our proposed fuzzy rule-based classification system. 


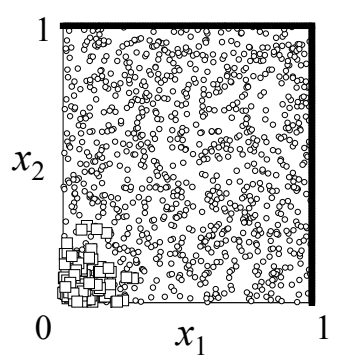

(a) $L=2$

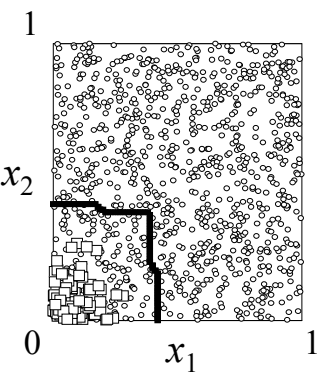

(c) $L=4$

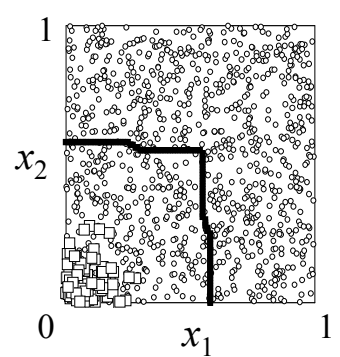

(b) $L=3$

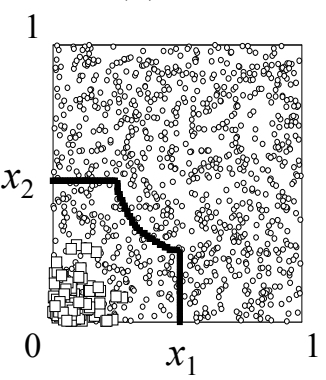

(d) $L=5$
Fig. 3. Classification boundaries by the proposed method.

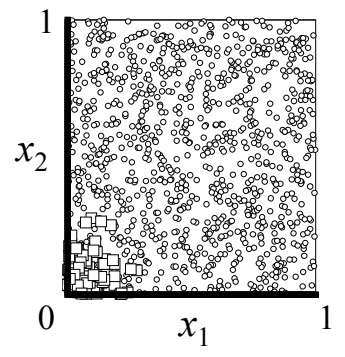

(a) $L=2$

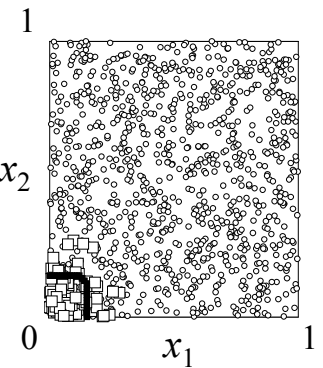

(c) $L=4$

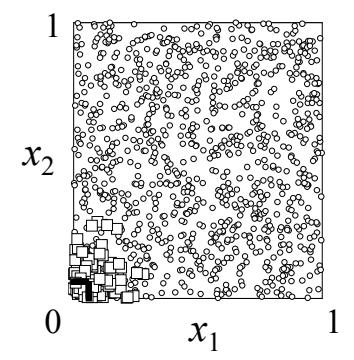

(b) $L=3$

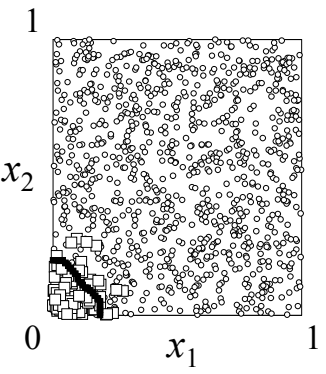

(d) $L=5$
Fig. 4. Classification boundaries by the conventional method.

\section{B. Medical diagnosis problems}

We then applied the fuzzy classifiers to two medical diagnosis problems: appendix and breast cancer (available from UCI Machine Learning Repository [19]). Table I lists the details of the datasets.

Since the classification priority is not given in the data sets in Table I, we specify it manually in order to generate a synthetic situation where the classification priority in the problems is given a priori. For medical diagnosis problems it is natural to assume that the classification of malignant patterns has higher priority than that of
TABLE I: Medical diagnosis problems.

\begin{tabular}{cccc}
\hline Data sets & Classes & Patterns & Attributes \\
\hline Appendix & 2 & 106 & 7 \\
Breast cancer & 2 & 683 & 9 \\
\hline
\end{tabular}

benign patterns. Thus, we assign the higher classification priority to malignant patterns and the lower classification priority to benign patterns (i.e. $r_{1}=1$ for malignant and $r_{2}=2$ for benign in Section III).

We specified the number of fuzzy partitions for each attribute as $L=3,4, \ldots, 15$. Since both the conventional and the proposed fuzzy rule-based classification systems are deterministic, we need to examine the performance of the two fuzzy rule-based classification systems just once. We show the classification results of the proposed method in Table II for the appendix diagnosis and in Table III for the breast cancer diagnosis. Tables IV and V shows the classification results obtained by the conventional fuzzy classifierfor comparison. All tables show the classification accuracies for the entire data set, for malignant patterns, and for benign patterns. Bold numbers in Tables II and III indicate that better results were obtained compared to the corresponding results obtained by the conventional fuzzy rule-based classification system in Tables IV and V.

We can see that $100 \%$ classification accuracy for malignant patterns with the higher classification priority was obtained by the proposed method for all the fuzzy partitions $L=3,4, \ldots, 15$ although the classification accuracy for benign patterns by the proposed method is not higher than that of the conventional method. This is because the classification priority of malignant cases is higher than that of benign ones. Thus, the proposed classifier focusses on the correct classification of malignant patterns more than that of benign patterns.

Next, we examined the performance of the proposed fuzzy rule-based classification systems on test data. Tenfold cross-validation was used where the data set is divided into ten disjoint subsets, one subset used as test data and the other nine subsets used as training patterns. A single trial is completed when all ten subsets have been used as test data once. Again, we examined the performance of the proposed and the conventional fuzzy rule-based classification systems with the fuzzy partitions $L=3,4, \ldots, 15$. Tables VI and Table VII show the classification results of our proposed classification systems while Tables VIII and IX give results for the conventional classifiers. Again. bold numbers show where superior results were obtained compared to the corresponding conventional fuzzy classification system.

It is apparent from Tables VI to IX that the classification priority is successfully incorporated in the proposed fuzzy rule-based classification systems not only for training data but also for test data. 
TABLE II: Classification accuracy by the proposed method for appendix training data.

\begin{tabular}{cccc}
\hline$L$ & Total & malignant & benign \\
\hline 3 & 37.74 & $\mathbf{1 0 0}$ & 22.35 \\
4 & 38.68 & $\mathbf{1 0 0}$ & 23.53 \\
5 & 64.15 & $\mathbf{1 0 0}$ & 55.29 \\
6 & 59.43 & $\mathbf{1 0 0}$ & 49.41 \\
7 & 75.47 & $\mathbf{1 0 0}$ & 69.41 \\
8 & 70.75 & $\mathbf{1 0 0}$ & 63.53 \\
9 & 86.79 & $\mathbf{1 0 0}$ & 83.53 \\
10 & 87.74 & $\mathbf{1 0 0}$ & 84.71 \\
11 & 89.62 & $\mathbf{1 0 0}$ & 87.06 \\
12 & $\mathbf{9 6 . 2 3}$ & $\mathbf{1 0 0}$ & 95.29 \\
13 & 94.34 & $\mathbf{1 0 0}$ & 92.94 \\
14 & $\mathbf{9 8 . 1 1}$ & $\mathbf{1 0 0}$ & 97.65 \\
15 & $\mathbf{9 8 . 1 1}$ & $\mathbf{1 0 0}$ & 97.65 \\
\hline
\end{tabular}

TABLE III: Classification accuracy by the proposed method for breast cancer training data.

\begin{tabular}{cccc}
\hline$L$ & Total & malignant & benign \\
\hline 3 & 80.82 & $\mathbf{1 0 0}$ & 45.19 \\
4 & 88.87 & $\mathbf{1 0 0}$ & 68.20 \\
5 & 90.04 & $\mathbf{1 0 0}$ & 71.55 \\
6 & 93.85 & $\mathbf{1 0 0}$ & 82.43 \\
7 & 96.19 & $\mathbf{1 0 0}$ & 89.12 \\
8 & 94.58 & $\mathbf{1 0 0}$ & 84.52 \\
9 & 94.14 & $\mathbf{1 0 0}$ & 83.26 \\
10 & 99.71 & $\mathbf{1 0 0}$ & 99.16 \\
11 & 94.29 & $\mathbf{1 0 0}$ & 83.68 \\
12 & 95.46 & $\mathbf{1 0 0}$ & 87.03 \\
13 & 98.10 & $\mathbf{1 0 0}$ & 94.56 \\
14 & 95.46 & $\mathbf{1 0 0}$ & 87.03 \\
15 & 95.46 & $\mathbf{1 0 0}$ & 87.03 \\
\hline
\end{tabular}

\section{Conclusions}

In this paper we proposed and examined the performance of fuzzy rule-based systems with classification priority for medical diagnosis problems. The assumption here is that the classification priority is given a priori for each class. The results of the computational experiments showed the effectiveness of the proposed classifier.

\section{REFERENCES}

[1] Lee. 1990, "Fuzzy Logic in Control Systems: Fuzzy Logic Controller Part I and Part II," IEEE Trans. Syst., Man, Cyberetics, Vol. 20:404-435.

[2] C.T. Leondes. 1999, "Fuzzy theory Systems," Techniques and Applications. Academic Press, San Diego, Vol.1-4, 1999.

[3] M. Sugeno. 1985, "An Introductory Survey of Fuzzy Control," Information Science, Vol. 30, No. 1/2:59-83.

[4] H. Ishibuchi, T. Nakashima, M. Nii, "Fuzzy IF-THEN Rules for Pattern Classification," Fuzzy If-Then Rules in Computational Intelligence: Theory and Applications, Kluwer Academic Publishers, pp. 267-295, May 2000.

[5] H. Ishibuchi, T. Nakashima, T. Morisawa, "Voting in Fuzzy Rule-Based Systems for Pattern Classification Problems," Fuzzy Sets and Systems, Vol.103, No.2, pp.223-238, April 1999.
TABLE IV: Classification accuracy by the conventional method for appendix training data.

\begin{tabular}{cccc}
\hline$L$ & Total & malignant & benign \\
\hline 3 & 79.25 & 0 & 98.82 \\
4 & 85.85 & 38.10 & 97.65 \\
5 & 88.68 & 47.62 & 98.82 \\
6 & 90.57 & 57.14 & 98.82 \\
7 & 92.45 & 61.90 & 100 \\
8 & 94.34 & 71.43 & 100 \\
9 & 93.40 & 66.67 & 100 \\
10 & 93.40 & 66.67 & 100 \\
11 & 94.34 & 71.43 & 100 \\
12 & 95.28 & 76.19 & 100 \\
13 & 96.23 & 80.95 & 100 \\
14 & 96.23 & 80.95 & 100 \\
15 & 95.28 & 76.19 & 100 \\
\hline
\end{tabular}

TABLE V: Classification accuracy by the conventional method for breast cancer training data.

\begin{tabular}{cccc}
\hline$L$ & Total & malignant & benign \\
\hline 3 & 95.46 & 98.42 & 89.96 \\
4 & 96.19 & 98.20 & 92.47 \\
5 & 96.49 & 98.65 & 92.47 \\
6 & 96.49 & 98.87 & 92.05 \\
7 & 96.63 & 98.42 & 93.31 \\
8 & 96.34 & 98.42 & 92.47 \\
9 & 96.34 & 98.65 & 92.05 \\
10 & 100 & 100 & 100 \\
11 & 96.63 & 99.10 & 92.05 \\
12 & 97.07 & 98.87 & 93.72 \\
13 & 98.24 & 99.55 & 95.82 \\
14 & 97.22 & 99.10 & 93.72 \\
15 & 97.22 & 99.10 & 93.72 \\
\hline
\end{tabular}

[6] H. Ishibuchi, T. Nakashima. 1999a, "Performance evaluation of fuzzy classifier systems for multi-dimensional pattern classification problems," IEEE Trans. on Syst., Man, Cybernetics, Part B Vol. 29:601-618.

[7] H. Ishibuchi, T. Nakashima. 1999b, "Improving the performance of fuzzy classifier systems for pattern classification problems with continuous attributes," IEEE Trans. on Industrial Electronics, Vol. 46, No. 6:1057-1068.

[8] H. Ishibuchi, K. Nozaki, N. Yamamoto, H. Tanaka. 1995, "Selecting fuzzy if-then rules for classification problems using genetic algorithms," IEEE Trans. on Fuzzy Systems, Vol. 3, No. 3:260-270.

[9] Y. Yuan, H. Zhang. 1996, "A genetic algorithms for generating fuzzy classification rules," Fuzzy Sets and Systems, Vol. 84, No. 1:1-19.

[10] R. O. Duda, P. E. Hart, D. G. Stork, Pattern Classification, Wiley Interscience, 2001.

[11] P. Domingos, "Metacost: A General Method for Making Classifiers Cost Sensitive," In Proceedings Fifth International Conference on Knowledge Discovery and Data Mining, 1999, pp. $155-164$.

[12] K. Nozaki, H. Ishibuchi, H. Tanaka. 1996, "Adaptive fuzzy rule-based classification systems," IEEE Trans. on Fuzzy Systems, Vol. 4, No. 3:238-250.

[13] G. J. Klir, B. Yuan. 1995, Fuzzy Sets and Fuzzy Logic, Prentice-Hall.

[14] H. Ishibuchi, K. Nozaki, H. Tanaka. 1992, "Distributed repre- 
TABLE VI: Classification accuracy by the proposed method for appendix test data.

\begin{tabular}{cccc}
\hline$L$ & Total & malignant & benign \\
\hline 3 & 36.73 & $\mathbf{9 5 . 0 0}$ & 22.36 \\
4 & 35.00 & $\mathbf{9 0 . 0 0}$ & 21.53 \\
5 & 63.27 & $\mathbf{8 5 . 0 0}$ & 57.78 \\
6 & 55.64 & $\mathbf{7 5 . 0 0}$ & 51.11 \\
7 & 67.09 & $\mathbf{6 6 . 6 7}$ & 67.50 \\
8 & 63.09 & $\mathbf{6 8 . 3 3}$ & 62.64 \\
9 & 77.37 & $\mathbf{6 3 . 3 3}$ & 81.25 \\
10 & 75.37 & $\mathbf{6 1 . 6 7}$ & 79.03 \\
11 & 73.73 & $\mathbf{5 3 . 3 3}$ & 78.75 \\
12 & 82.18 & $\mathbf{5 8 . 3 3}$ & 88.47 \\
13 & 78.18 & $\mathbf{3 8 . 3 3}$ & 87.22 \\
14 & 83.91 & $\mathbf{6 0 . 0 0}$ & 90.69 \\
15 & 81.09 & $\mathbf{5 3 . 3 3}$ & 88.33 \\
\hline
\end{tabular}

TABLE VII: Classification accuracy by the proposed method for breast test data.

\begin{tabular}{cccc}
\hline$L$ & Total & malignant & benign \\
\hline 3 & 81.12 & $\mathbf{9 9 . 7 8}$ & 46.82 \\
4 & 89.17 & $\mathbf{9 9 . 5 7}$ & 69.31 \\
5 & 89.61 & $\mathbf{9 8 . 6 6}$ & 73.29 \\
6 & 92.83 & $\mathbf{9 8 . 6 6}$ & 81.84 \\
7 & 94.89 & $\mathbf{9 7 . 7 1}$ & 89.42 \\
8 & 93.27 & $\mathbf{9 8 . 2 2}$ & 83.84 \\
9 & 93.13 & $\mathbf{9 8 . 1 9}$ & 83.76 \\
10 & 97.08 & $\mathbf{9 6 . 8 0}$ & 97.36 \\
11 & 92.98 & $\mathbf{9 8 . 4 3}$ & 83.02 \\
12 & 93.42 & $\mathbf{9 7 . 7 4}$ & 85.20 \\
13 & 94.59 & $\mathbf{9 6 . 3 4}$ & 91.11 \\
14 & 93.27 & $\mathbf{9 7 . 9 7}$ & 84.45 \\
15 & 93.27 & $\mathbf{9 7 . 9 7}$ & 84.45 \\
\hline
\end{tabular}

sentation of fuzzy rules and its application to pattern classification," Fuzzy Sets and Systems, Vol. 52, No. 1:21-32.

[15] M. Grabisch. 1996, "The representation of importance and interaction of features by fuzzy measures," Pattern Recognition Letters, Vol. 17:567-575.

[16] M. Grabisch, F. Dispot. 1992 "A comparison of some methods of fuzzy classification on real data," Proc. of 2nd Intl. Conf. on Fuzzy Logic and Neural Networks, 659-662.

[17] M. Grabisch, and J.-M. Nicolas. 1994, "Classification by fuzzy integral: performance and tests," Fuzzy Sets and Systems, Vol. 65 , No. 2/3:255-271.

[18] H. Ishibuchi, T. Nakashima. 2001, "The Effect of rule weights in fuzzy rule-based classification systems," IEEE Trans. on Fuzzy Systems, Vol. 9, No. 4:506-515.

[19] D.J. Newman, S. Hettich, C.L. Blake, and C.J. Merz, "UCI Repository of machine learning databases,"1998.
TABLE VIII: Classification accuracy by the conventional method for Appendix (Test data).

\begin{tabular}{cccc}
\hline$L$ & Total & malignant & benign \\
\hline 3 & 78.46 & 0 & 97.78 \\
4 & 84.09 & 30.00 & 97.78 \\
5 & 86.82 & 40.00 & 97.64 \\
6 & 84.00 & 40.00 & 94.17 \\
7 & 83.09 & 35.00 & 94.17 \\
8 & 85.09 & 55.00 & 93.06 \\
9 & 87.73 & 60.00 & 95.28 \\
10 & 85.00 & 50.00 & 94.17 \\
11 & 84.18 & 50.00 & 93.06 \\
12 & 86.00 & 55.00 & 94.17 \\
13 & 84.09 & 35.00 & 95.56 \\
14 & 87.82 & 55.00 & 96.67 \\
15 & 83.09 & 50.00 & 91.94 \\
\hline
\end{tabular}

TABLE IX: Classification accuracy by the conventional method for Breast W (Test data).

\begin{tabular}{cccc}
\hline$L$ & Total & malignant & benign \\
\hline 3 & 95.18 & 98.22 & 89.65 \\
4 & 95.77 & 97.50 & 92.39 \\
5 & 95.47 & 97.74 & 91.08 \\
6 & 95.62 & 97.50 & 91.91 \\
7 & 96.06 & 97.50 & 93.20 \\
8 & 95.61 & 97.50 & 91.82 \\
9 & 95.76 & 97.97 & 91.51 \\
10 & 97.23 & 96.80 & 98.02 \\
11 & 95.47 & 98.22 & 90.66 \\
12 & 95.47 & 97.31 & 92.03 \\
13 & 95.03 & 96.34 & 92.39 \\
14 & 95.61 & 97.74 & 91.38 \\
15 & 95.61 & 97.74 & 91.38 \\
\hline
\end{tabular}

